\title{
Detecting Unattended Stimuli Depends on the Phase of Prestimulus Neural Oscillations
}

\author{
(A)Anthony M. Harris, ${ }^{1}$ PPaul E. Dux, ${ }^{2}$ and ${ }^{\circ}$ Jason B. Mattingley ${ }^{1,2}$ \\ ${ }^{1}$ Queensland Brain Institute and ${ }^{2}$ School of Psychology, The University of Queensland, St. Lucia 4072, Australia
}

\begin{abstract}
Neural oscillations appear important for perception and attention processes because stimulus detection is dependent upon the phase of 7-11 Hz oscillations before stimulus onset. Previous work has examined stimulus detection at attended locations, but it is unknown whether unattended locations are also subject to phasic modulation by ongoing oscillatory activity, as would be predicted by theories proposing a role for neural oscillations in organizing general neural processing. Here, we recorded brain activity with EEG while human participants of both sexes detected brief visual targets preceded by a spatial cue and determined whether performance for cued (attended) and uncued (unattended) targets was influenced by oscillatory phase across a range of frequencies. Detection of both attended and unattended targets depended upon an $\sim 5 \mathrm{~Hz}$ theta rhythm and an $\sim 11-15 \mathrm{~Hz}$ alpha rhythm. Critically, detection of unattended stimuli was more strongly modulated by the phase of theta oscillations than was detection of attended stimuli, suggesting that attentional allocation involves a disengagement from ongoing theta sampling. There was no attention-related difference in the strength of alpha phase dependence, consistent with a perceptual rather than attentional role of oscillatory phase in this frequency range. These results demonstrate the importance of neural oscillations in modulating visual processing at both attended and unattended locations and clarify one way in which attention may produce its effects: through disengagement from low-frequency sampling at attended locations.
\end{abstract}

Key words: alpha; attention; neural oscillations; perception; phase; theta

\section{Significance Statement}

Past work on the interaction between oscillatory phase and neural processing has shown the involvement of posterior $\sim 7-11 \mathrm{~Hz}$ oscillations in visual processing. Most studies, however, have presented stimuli at attended locations, making it difficult to disentangle frequencies related to attention from those related to perception. Here, we compared the oscillatory frequencies involved in the detection of attended and unattended stimuli and found that $\sim 11-15 \mathrm{~Hz}$ oscillations were related to perception independently of attention, whereas $\sim 5 \mathrm{~Hz}$ oscillations were more prominent for the detection of unattended stimuli. This work demonstrates the importance of neural oscillations for mediating stimulus processing at both attended and unattended locations and clarifies the different oscillatory frequencies involved in attention and perception.

\section{Introduction}

Rhythmic fluctuations in neural activity are ubiquitous throughout the brain (Buzsáki, 2006). Such oscillatory activity has been suggested to play a critical role in neural processing (Buzsáki and Draghun, 2004) and, by extension, in cognitive and perceptual

\footnotetext{
Received Oct. 18, 2017; revised Jan. 31, 2018; accepted Feb. 12, 2018.

Author contributions: A.M.H., P.E.D., and J.B.M. designed research; A.M.H. performed research; A.M.H., P.E.D., and J.B.M. analyzed data; A.M.H., P.E.D., and J.B.M. wrote the paper.

This work was supported by the ARC Centre of Excellence for Integrative Brain Function (ARC Centre Grant (E140100007). J.B.M. was supported by an ARC Australian Laureate Fellowship (FL110100103). PED was supported by an ARC Future Fellowship (FT120100033). We thank Susan Travis for helpful comments on an earlier version of this manuscript.

The authors declare no competing financial interests.

Correspondence should be addressed to Anthony M. Harris, Queensland Brain Institute, The University of Queensland, St. Lucia QLD 4072, Australia. E-mail: anthmharris@gmail.com.

DOI:10.1523/JNEUROSCI.3006-17.2018

Copyright $\odot 2018$ the authors $\quad 0270-6474 / 18 / 383092-10 \$ 15.00 / 0$
}

processes (Başar et al., 2001). Studies using magnetoencephalography (MEG) and electroencephalography (EEG) have shown that the position within an oscillatory cycle (the phase, e.g., peak vs trough) predicts behavioral outcomes at key times in the prestimulus period (VanRullen, 2016b). In particular, studies presenting visual stimuli at perceptual threshold have shown that stimulus detection at attended locations fluctuates with the phase of 7-11 Hz oscillations (Busch et al., 2009; Mathewson et al., 2009; Busch and VanRullen, 2010; Sherman et al., 2016). However, few studies have endeavored to differentiate phasic influences affecting detection of attended stimuli from those affecting unattended stimuli (but see Busch and VanRullen, 2010). Here, we compare the phase dependence of perception between attended and unattended locations in a spatially cued visual detection paradigm.

Most studies examining the influence of prestimulus oscillatory phase on perception have presented stimuli at a known cen- 


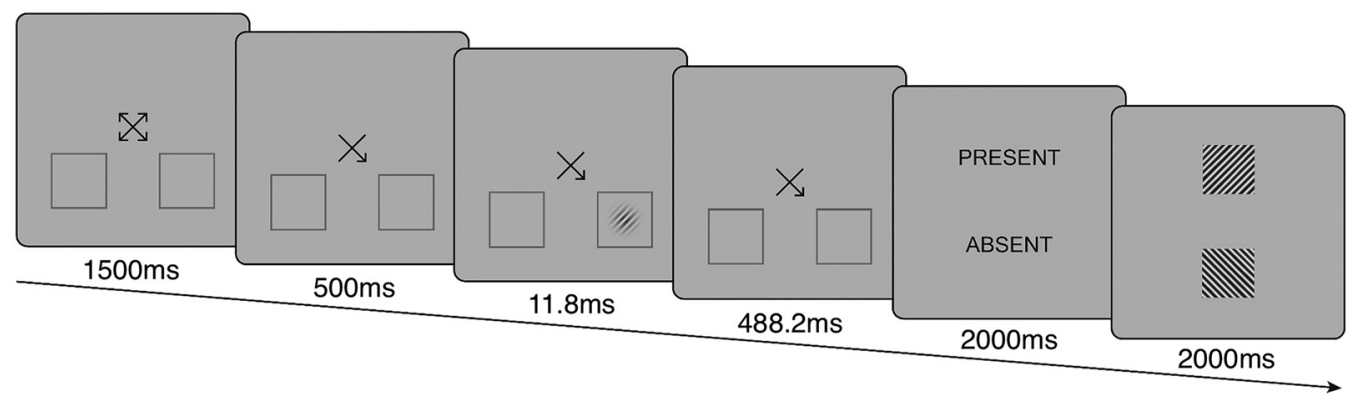

Figure 1. Schematic stimuli (not to scale). Participants fixated centrally, were cued to attend to one of the two boxes, and were required to report the presence/absence and orientation of a set of oriented lines that could appear in either box.

tral or peripheral location (Busch et al., 2009; Mathewson et al., 2009; Fiebelkorn, Snyder et al., 2013; Sherman et al., 2016). In an exception to this trend, Busch and VanRullen (2010) presented low-contrast targets at cued and uncued locations and binned trials based on the phase of oscillations in the pretrial period. They found that detection of cued stimuli decreased monotonically with increasing difference from the optimal phase bin of a prestimulus $\sim 7 \mathrm{~Hz}$ oscillation at frontocentral EEG electrodes. There was no effect of prestimulus phase on the detection of uncued stimuli, suggesting that stimulus processing at unattended locations is not subject to phasic modulation by ongoing oscillations. However, consideration of the analysis used by Busch and VanRullen (2010) suggests another possibility. It is well established that striate and extrastriate visual cortex is retinotopically organized, with lateralized stimuli producing responses primarily in contralateral visual regions (Zeki, 1978). This is important because Busch and VanRullen (2010) did not contralateralize their data before analysis. Therefore, their design may not have been sensitive to detecting phase dependence at lateral electrode sites, where early attentional effects are typically observed (Eimer, 2014).

Some studies have sought to identify oscillations involved in stimulus processing by examining rhythmic fluctuations in the temporal profile of behavioral responses. These studies have consistently demonstrated an $\sim 4 \mathrm{~Hz}$ rhythm in the perception of stimuli at unattended locations (Fiebelkorn, Saalmann, and Kastner, 2013; Dugué et al., 2016) or under conditions of distributed attention (Landau and Fries, 2012). Although these studies do not measure neural activity and thus cannot provide a definitive link between the phase of endogenous neural oscillations and perception, they do indicate that rhythmic processes are involved at some stage during stimulus processing. Complementing these results, a recent MEG study (Landau et al., 2015) showed a link between prestimulus $4 \mathrm{~Hz}$ neural activity (specifically, thetagamma cross-frequency coupling) and stimulus detection under conditions of distributed attention. It remains unknown whether this theta phase dependence is modulated when spatial attention is allocated to a specific location, consistent with the results of the above behavioral studies.

Here, we investigated whether prestimulus phase at contralateral electrode sites modulates stimulus detection outside the current focus of spatial attention. If unattended locations are not subject to phasic sampling, then we expect no relationship between prestimulus phase and uncued-target detection (Busch and VanRullen, 2010). If unattended locations are subject to phasic sampling, then this could present in two ways. Phase dependence of uncued-target detection might occur at the same frequencies and with the same strength as for cued stimuli, indi- cating a sampling process that is independent of focused spatial attention. Alternatively, phasic modulation of uncued-target detection may occur at distinct frequencies or with different strength compared with cued stimuli, indicating a sampling process modulated by attention. Our results supported the final possibility, with stronger $\sim 5 \mathrm{~Hz}$ modulation for uncued stimuli compared with cued stimuli.

\section{Materials and Methods \\ Participants}

We tested 36 participants ( 26 females, mean age 21.97 years, $S D=1.66$ years); of these, nine participants were excluded for making eye movements on $>25 \%$ of trials (criterion decided a priori). One additional participant was excluded for reporting the target as absent on $>85 \%$ of trials, leaving insufficient target-present trials for analysis. This left 26 participants in the final analyzed dataset (20 females, mean age 22.08 years, $\mathrm{SD}=1.74$ years). Participants all self-reported as right handed and had normal or corrected-to-normal vision, provided written informed consent, and were reimbursed at a rate of $\$ 10 / \mathrm{h}$ for their participation. The Human Research Ethics Committee at The University of Queensland approved the study.

\section{Stimuli and procedure}

The stimuli and behavioral paradigm in this experiment were modeled on those of Wyart and Tallon-Baudry (2008). Participants were presented with a fixation display containing a central black fixation cross (RGB: $000 ; 0.42^{\circ} \times 0.42^{\circ}$ ) with an arrowhead at the end of each arm of the cross (arrowhead length: $0.15^{\circ}$ ). In the lower visual hemifield were two dark gray placeholder boxes (RGB: 192192 192; 2.2 $\times 2.2^{\circ}$; line thickness $=2$ pixels) placed $6^{\circ}$ horizontally from fixation and $3^{\circ}$ below the horizontal midline (Fig. 1). All displays were presented on a midgray background (RGB: 128128 128). After $1500 \mathrm{~ms}$, three of the arrowheads on the central cross disappeared, leaving one arrow pointing to one of the two boxes. This cue display was present for $500 \mathrm{~ms}$. After this, the target appeared for $11.8 \mathrm{~ms}$ on target-present trials or the screen remained unchanged for this duration on target-absent trials. The target was a Gaussian-windowed circular Gabor patch (diameter: $2^{\circ}$; spatial frequency: 5 cycles $/^{\circ}$ ) oriented at 1 of 8 evenly spaced angles from $20^{\circ}$ to $160^{\circ}$ and located centrally in one of the placeholder boxes. During the experiment, the maximum contrast value for the target was set at each participant's individual contrast threshold for $50 \%$ correct detection. Critically, this procedure for titrating the contrast value of targets was undertaken separately for cued and uncued trials, so that performance was matched for the two trial types in terms of detection and discrimination. Color bit stealing (Tyler, 1997) was used to produce a finer resolution of contrast values than would otherwise be available. Contrast levels were titrated before each experimental session, as described below. On cued trials ( $60 \%$ of trials), the target appeared in the box indicated by the cue. On uncued trials (30\% of trials), the target appeared in the opposite box. On target-absent trials ( $10 \%$ of trials), no target was presented. After the target period, the cue display remained present for a further $488.2 \mathrm{~ms}$. 
This was followed by the detection probe, in which the words "present" and "absent" were displayed in 24 point Arial font at central locations $2^{\circ}$ above and below fixation and the positons were counterbalanced across trials. After $2000 \mathrm{~ms}$, the detection probe was replaced by the orientation probe in which two large square gratings $\left(4^{\circ} \times 4^{\circ} ; 20 \%\right.$ contrast; spatial frequency $=5 \mathrm{cycles} /{ }^{\circ}$ ) were centered $3^{\circ}$ above and below fixation on the vertical midline for $2000 \mathrm{~ms}$. The orientation test gratings were displayed at relatively low contrast to minimize any potential visual aftereffects they might have caused and were positioned so as not to overlap the target locations. The orientation of one test grating matched the target orientation (counterbalanced to be the top or bottom grating equally often) and the other orientation was $\pm 60^{\circ}$ from the target orientation. On target-absent trials, an orientation was selected at random and the second orientation differed from this by $\pm 60^{\circ}$. After the orientation probe, the next trial began with the fixation display. No feedback on accuracy or response time was given. Throughout the experiment, participants' brain activity was recorded with EEG, as described in detail below.

On each trial, participants were asked to fixate the central cross and direct their attention to the cued box without blinking or moving their eyes. During the present/absent probe, they were asked to select "present" if they felt they had seen the target and to select "absent" otherwise. In the orientation probe, participants were asked to select the grating orientation that matched that of the target as accurately as possible regardless of whether they actually detected the target and asked to guess in the case of undetected targets. Participants responded by pressing the up or down arrow keys with their right hand (for the top or bottom options, respectively) on a standard USB keyboard.

Participants performed $2 \times 2.5 \mathrm{~h}$ sessions of the task, each on separate days. In each session, participants were given written and verbal instructions and were then shown 10 example trials to familiarize themselves with the task. In the first five practice trials, the target was presented at $80 \%$ contrast to introduce participants to the structure of the task. After the first five practice trials, participants were informed that, in the actual task, the target would be more difficult to see. They were then exposed to five more practice trials in which the target was presented at $40 \%$ contrast to provide a more realistic example of the true task conditions. Each of the five trial practice blocks contained two cued-target trials, two uncuedtarget trials, and one target-absent trial.

After this introduction, participants completed the target threshold titration block, which consisted of 250 trials of the task described above in which the contrast of the targets was adjusted from trial to trial until participants produced a $50 \%$ rate of target detection. Contrast titration was performed using the QUEST algorithm for threshold estimation (Watson and Pelli, 1983). Two independent QUEST staircases were used for each of the cued-target and uncued-target conditions. Contrast levels began at $50 \%$ and were adjusted up or down based on the reports of the participant to achieve a $50 \%$ detection rate in each of the two different cue conditions. To provide more stable estimates, the estimated thresholds from the two cued-target staircases were averaged at the end of titration, as were those from the two uncued-target staircases.

Next, participants completed the test phase, which was identical to the titration procedure except that targets were now presented at the individual's estimated contrast thresholds. Participants completed 250 test trials per session. In both titration and test blocks, participants were given a short break every 50 trials.

Stimuli were presented on a gamma-corrected NEC AccuSync 120 CRT monitor with a $1024 \times 786$ display resolution and an $85 \mathrm{~Hz}$ refresh rate. Stimulus presentation was controlled using the Psychophysics Toolbox 3 extension (Brainard, 1997; Kleiner et al., 2007; RRID:SCR_002881) for MATLAB (The MathWorks; RRID:SCR_001622), running under Windows XP, Service Pack 3. Viewing distance was maintained at $57 \mathrm{~cm}$ using a chinrest.

\section{Experimental design and statistical analysis: behavior}

Behavioral data were analyzed with repeated-measures ANOVAs (see the Results section for a description of the factors and dependent variable for each test). All ANOVAs were run using JASP (version 0.8.5; RRID: SCR_015823).

\section{EEG recording}

Continuous EEG data were recorded using an Active Two system (BioSemi) digitized at $1024 \mathrm{~Hz}$ with 24-bit A/D conversion. Sixty-four active scalp $\mathrm{Ag} / \mathrm{AgCl}$ electrodes were arranged according to the international standard 10-10 system for electrode placement (Oostenveld and Praamstra, 2001) using a nylon head cap. As per the BioSemi system design, the Common Mode Sense and Driven Right Leg electrodes served as the ground and all scalp electrodes were referenced to the Common Mode Sense electrode during recording. Eye movements were monitored using bipolar horizontal EOG electrodes placed at the outer canthi of each eye and bipolar vertical EOG electrodes were placed above and below the left eye.

\section{Experimental design and statistical analysis: EEG}

Offline EEG data analysis was performed using EEGLAB (Delorme and Makeig, 2004; RRID:SCR_007292) and custom MATLAB scripts (except where noted). The data were downsampled to $512 \mathrm{~Hz}$, high-pass filtered at $0.1 \mathrm{~Hz}$ using the EEGLAB function firfilt(), and referenced to the average of all scalp electrodes. Noisy channels, identified by visual inspection of the data, were replaced by a spherical spline interpolation of the voltages recorded at neighboring scalp electrodes. The data were then preliminarily segmented into $7 \mathrm{~s}$ epochs spanning the duration of each trial and baseline corrected between 400 and $200 \mathrm{~ms}$ before cue onset for the purpose of inspecting the data. Because large neural events may influence the phase estimates at surrounding times, epochs containing excessive noise or contaminated by eye blinks, saccades, or other muscle activity that occurred at any time within the period from onset of the fixation stimulus until the appearance of the detection probe after the target were excluded from further analysis; this led to an average loss of $3.2 \%$ of all trials. To ensure that phase estimates were not influenced by neural responses to the target, we excluded from analysis the pretarget period in which the phase estimation procedure could have been influenced by target-related activity (the temporal smearing region; see below for further details).

We next applied a surface Laplacian to the data using the spherical spline method of Perrin et al. (1989) as implemented in the laplacian_ perrinX() function from Cohen (2014). The surface Laplacian acts to attenuate low spatial frequencies from the topographical distribution of EEG data, reducing the impact of volume conduction on measured potentials. It was used here to allow us to estimate the phase of prestimulus oscillations at scalp electrodes showing the first early visual responses while minimizing the influence of volume-conducted activity from other cortical sources on phase estimates. The data resulting from the surface Laplacian were used for all EEG analyses. Repeating the event-related potential (ERP) analyses and time-frequency power analyses without applying a surface Laplacian to the data produced qualitatively identical results.

For ERP analyses, the data were re-epoched to the onset of the relevant event (target-evoked and cue-evoked ERPs were analyzed separately, as described below) and were baseline corrected by subtracting from all time points the mean of the period from $100 \mathrm{~ms}$ before the event until event onset. EEG responses were contralateralized relative to the location of the target by swapping each left side electrode with its corresponding right side electrode on trials in which the target appeared to the left of fixation. The data from trials in which the target appeared to the right of fixation remained unchanged. Therefore, data were combined from the electrode contralateral to the target's location regardless of which location the target appeared in. ERPs were calculated by averaging all trials in the relevant combination of cue and detection conditions for each session and then averaged across sessions. Significance tests for targetevoked ERPs were performed at every electrode at every time point from 0 to $500 \mathrm{~ms}$, controlling for multiple comparisons with cluster-based permutation tests (Groppeet al., 2011). The cluster-based permutation test was 2 -tailed with a $p=0.01$ threshold for inclusion in a cluster and used 5000 permutations. Analyses were terminated at $500 \mathrm{~ms}$ after target onset so as not to include responses evoked by the detection probe in the analysis.

For our pretarget analyses, we chose to examine the posterior electrodes that showed the largest post-target evoked response. Therefore, all 
other EEG analyses in addition to the target-evoked ERPs described above were performed at posterior electrodes that showed the largest target-evoked negativity in the ERP response for each individual (from the set $\{\mathrm{P} 1 / 2, \mathrm{P} 3 / 4, \mathrm{P} 5 / 6, \mathrm{P} 7 / 8, \mathrm{P} 9 / 10, \mathrm{PO} 3 / 4, \mathrm{PO} 7 / 8, \mathrm{O} 1 / 2\})$, collapsed across all trials, and averaged across the period from 175 to $225 \mathrm{~ms}$ after target onset (hereafter referred to as each individual's focal electrodes). This time period was selected for averaging because it encompassed the time period of the maximum significant difference between detected and undetected targets in the target-locked ERP analysis, as described in detail in the Results. A separate set of focal electrodes was selected for each session to account for between-session variability in the topography of responses due, for example, to minor variations in EEG cap placement. For the majority of participants, the focal electrodes were PO7/8 (session 1: $18 / 26$ participants, session $2: 17 / 26$ participants). The remainder of the participants had their focal electrodes at locations surrounding these (session 1: P3/4: 1 participant; P5/6: 1 participant; P7/8: 1 participant; P9/10: 2 participants; $\mathrm{PO} / 4$ : 2 participants; O1/2: 1 participant; session 2: P1/2: 1 participant; P3/4: 2 participants; P5/6: 1 participant; P7/8: 2 participants; PO3/4: 2 participants; O1/2: 1 participant).

To ensure that pretarget differences in phase dependence at particular times and frequencies were not driven by differences in the cue-evoked ERP, we compared the pretarget cue-evoked ERPs between trials in which the target was detected and those in which it was not. This analysis was performed at each participant's focal electrodes contralateral to the subsequent target for each session before averaging the ERPs across sessions. This was to provide an adequate control for the phase dependence analysis that was also performed at contralateral focal electrodes. We compared detected-target with undetected-target trials separately for cued and uncued conditions because these were the comparisons that we made in the phase dependence analysis. For this analysis, we used false discovery rate (FDR)-corrected $t$ tests (Benjamini and Hochberg, 1995) at each time point from cue onset to target onset, but we also report the uncorrected results to provide a maximally informative control.

For time-frequency analyses, the data were epoched from 1500 before to $1500 \mathrm{~ms}$ after target onset. Time-frequency analyses were performed by convolving each trial epoch with 3-cycle complex Morlet wavelets (Tallon-Baudry and Bertrand, 1999; Cohen, 2014) at 39 linearly spaced frequencies from 2 to $40 \mathrm{~Hz}$. Three-cycle wavelets were used to provide reasonable frequency precision while not contaminating too much of the pretrial period with activity that may have been smeared from poststimulus responses. Power and phase were extracted from the complex result of the wavelet transform.

Power data were baseline corrected against the period from -800 to $-600 \mathrm{~ms}$ pretarget ( -300 to -100 precue) using decibel conversion. Analysis of lateralized oscillatory power in the cue period was performed across time and frequency at each individual's focal electrodes, comparing power contralateral to the cued hemifield with that to the ipsilateral cued hemifield. For this analysis, we again used FDR-controlled $t$ tests (Benjamini and Hochberg, 1995) at all frequencies and all times from $-500 \mathrm{~ms}$ (cue onset) to $0 \mathrm{~ms}$ (target onset). Although correction against a pretrial baseline is not strictly necessary for analysis of contralateralipsilateral difference scores, it was used here to allow visualization of full scalp topographies of the power data. Analysis of decibel-converted differences between contralateral and ipsilateral focal electrodes with no pretrial baseline correction produced very similar results.

Analysis of phase dependence of perception was performed using the phase opposition sum (POS) metric (Drewes and VanRullen, 2011; Dugué, Marque, and VanRullen, 2011; VanRullen, 2016a). This metric quantifies the extent to which two groups of trials (e.g., detected targets and undetected targets) are associated with phase clustering at differing average phase angles at a particular time and frequency. Phase clustering is quantified by taking the magnitude of the vector produced by taking the circular mean of unit length vectors at measured phase angles for a particular time point across trials. The POS metric capitalizes on the fact that, if two conditions show strong phase clustering at opposite phase angles, then their individual phase-clustering values should exceed the phase-clustering value produced by all trials from both conditions combined. If these conditions are met, then the POS will be high. In comparison, if the two conditions do not show strong phase clustering at a particular time point or if they do but the phases cluster at approximately the same phase angles in both conditions, then the POS will be low. Under these conditions, the separate phase-clustering values of the two conditions will be approximately equivalent to or exceeded by the phase clustering of the conditions combined (see VanRullen, 2016a, for further description and diagrammatic examples). The POS metric was chosen instead of other available methods such as the circular Watson-Williams test because the uncued condition yielded a total of 150 trials across both sessions and VanRullen (2016a) found that the POS metric provided the best statistical power with $<200$ trials relative to other phase opposition metrics. To maximize trial numbers for this analysis, trials from sessions 1 and 2 were combined before the POS calculation.

Group-level statistics for the POS measure were assessed by computing individual-level $p$-values via $z$-scored permutation tests (VanRullen, $2016 \mathrm{a})$ with 5000 permutations and combining $p$-values across individuals using Stouffer's method (Stouffer et al., 1949), which transforms individual $p$-values into $z$-scores, combines them across participants, and converts the resulting $z$-score to a combined probability. The implementation of this process in MATLAB produced a combined $p$-value of 0 for input $p$-values $<10^{-16}$. Therefore, input $p$-values below this value were changed to $10^{-16}$ before $p$-value combination with Stouffer's method. The subsequent $p$-values were adjusted using FDR correction for multiple comparisons. The POS analysis, $z$-scored permutation tests, and $p$-value combination were performed using MATLAB code that accompanies VanRullen (2016a). POS analyses were performed from $-500 \mathrm{~ms}$ (cue onset) until the first time point at which phase values could have been influenced by posttarget activity (i.e., the temporal-smearing boundary). Because we used 3-cycle wavelets for the time-frequency decomposition, any time points within 1.5 cycles of target onset could have been influenced by posttarget activity. Therefore, the temporal-smearing boundary necessarily differed as a function of frequency (e.g., 1.5 cycles at $4 \mathrm{~Hz}$ yields a smearing width of $375 \mathrm{~ms}$, whereas 1.5 cycles at $10 \mathrm{~Hz}$ produces a smearing width of $150 \mathrm{~ms}$ ). Activity in the period between the temporal-smearing boundary and target onset was excluded from the POS analysis.

As planned a priori, we collapsed across sessions 1 and 2 for all EEG analyses. As a post hoc check, we repeated all behavioral analyses with session (1 vs 2) as an additional factor and found that session did not interact with any other factor. The only significant effect of session was a main effect in the analysis of contrast thresholds. Therefore, session is included in our reporting of the contrast threshold analysis.

\section{Results}

\section{Behavioral}

A 2 (cue condition: cued-target, uncued-target) $\times 2$ (session: 1 , 2) repeated-measures ANOVA on the contrast thresholds determined via titration and used in the main experiment revealed a significant main effect of cue condition $\left(F_{(1,25)}=16.72, p<\right.$ $\left.0.001, \eta^{2}=0.40\right)$, demonstrating that participants' contrast thresholds were lower at the cued location than at the uncued location (Fig. 2A), consistent with participants attending to the cued location and previous demonstrations of attention's influence on contrast thresholds (Pestilli and Carrasco, 2005). There was also a significant main effect of session $\left(F_{(1,25)}=13.12, p=\right.$ $\left.0.001, \eta^{2}=0.34\right)$, reflecting a decrease in thresholds due to practice from the first to the second session. There was no significant interaction $\left(F_{(1,25)}=1.03, p=0.321, \eta^{2}=0.04\right)$. Figure $1 B$ shows that the contrast thresholds in this experiment were relatively high $(\sim 27.5 \%)$ due to the extremely brief duration of the target stimuli (11.8 ms).

Participants reported stimuli as present on $56 \%$ of cued-target trials and on $54 \%$ of uncued-target trials (Fig. 2B). These percentages were not significantly different $\left[t_{(25)}=0.97, p=\right.$ 0.343 , Cohen's $d=0.19$, corrected for the dependence between means with Morris and DeShon's (2002) equation 8, allowing comparison with Cohen's d from between-groups tests]. As expected, a 2 (reported perception: present, absent) $\times 2$ (cue con- 
A

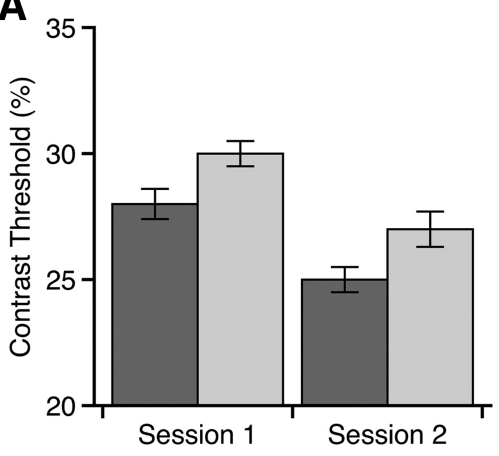

B

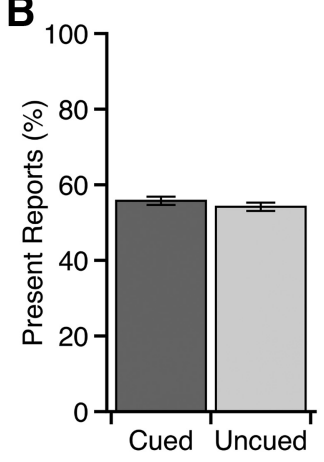

C

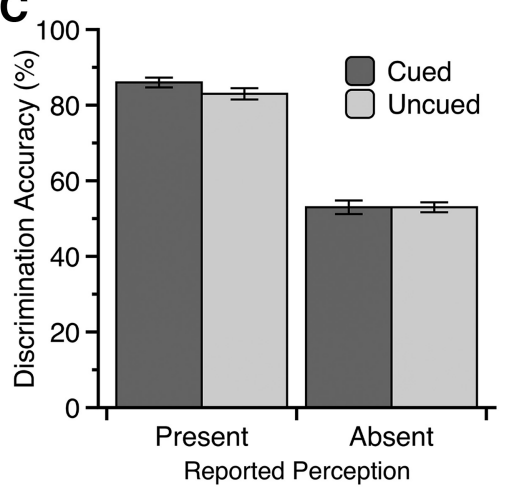

Figure 2. Behavioral results. $\boldsymbol{A}$, Contrast thresholds for $50 \%$ detection as estimated by QUEST (Watson and Pelli, 1983). These thresholds show the effect of attention. $\boldsymbol{B}$, Percentage of trials in which participants reported perceiving the target in the main experiment. $C$, Performance on the orientation discrimination task in the main experiment showing the effect of awareness. Error bars indicate within-participant SEM (Cousineau, 2005; Morey, 2008).

dition: cued-target, uncued-target) repeated-measures ANOVA on discrimination accuracy (Fig. 2C) revealed that participants were significantly more accurate in judging the orientation of the target on trials in which they reported perceiving it than on trials in which they $\operatorname{did} \operatorname{not}\left(F_{(1,25)}=251.48, p<0.001, \eta^{2}=0.91\right)$. There was no significant main effect of cue condition $\left(F_{(1,25)}=\right.$ $\left.1.22, p=0.280, \eta^{2}=0.05\right)$ and no significant interaction $\left(F_{(1,25)}=\right.$ $\left.2.05, p=0.164, \eta^{2}=0.08\right)$. False-positive rates were low $(\mathrm{M}=10 \%$ of target-absent trials, $\mathrm{SD}=12 \%$ ). Average sensitivity was positive $\left(\mathrm{d}^{\prime}=1.75, \mathrm{SD}=0.16\right)$ and was significantly different from zero $\left(t_{(25)}=11.54, p<0.001\right.$, Cohen's $\left.\mathrm{d}=2.31\right)$. Average bias was conservative $(\log \beta=1.56, \mathrm{SD}=0.28)$ and significantly different from zero $\left(t_{(25)}=5.85, p<0.001\right.$, Cohen's $\left.\mathrm{d}=1.17\right)$.

\section{ERPs}

Cluster permutation analysis comparing contralateralized ERP responses between detected targets and undetected targets collapsed across cue condition revealed a significantly more negative response when targets were detected than when they went undetected at a cluster of eight contralateral posterior electrodes (Fig. 3 , top) from 165 to $\sim 240 \mathrm{~ms}$ after stimulus onset, peaking at 203 $\mathrm{ms}$, at contralateral electrodes $\mathrm{PO} 7 / 8$. This negativity was accompanied by a small but significant positive difference at six ipsilateral parieto-occipital electrodes from 174 to $227 \mathrm{~ms}$ and maximal at ipsilateral electrodes PO3/4. These early effects were followed by widespread differences such that responses to detected targets were significantly more positive than those to undetected targets, beginning at $\sim 260 \mathrm{~ms}$ after target onset and persisting until the onset of the detection probe at $500 \mathrm{~ms}$ (data not shown). Comparing ERPs between detected and undetected targets, separately for cued-target trials and uncued-target trials produced very similar results to those described above (Fig. 3, middle and bottom, respectively). Comparing detected versus undetected difference scores between cued-target and uncued-target trials produced no significant clusters, suggesting no interaction between cue condition and detection on ERPs.

Our phase analysis, described below, examined oscillatory phase in the pretarget period and thus overlapped with the period of cue-evoked ERPs. To ensure that any finding of phase dependence cannot be attributed to differential cue-evoked ERPs for detected and undetected trials, we compared ERPs for these two trial types from $-500 \mathrm{~ms}$ (cue onset) until $0 \mathrm{~ms}$ (target onset) at each individual's focal electrode contralateral to the upcoming target location. Repeated-measures $t$ tests at every time point corrected for multiple comparisons by controlling the FDR (Ben-
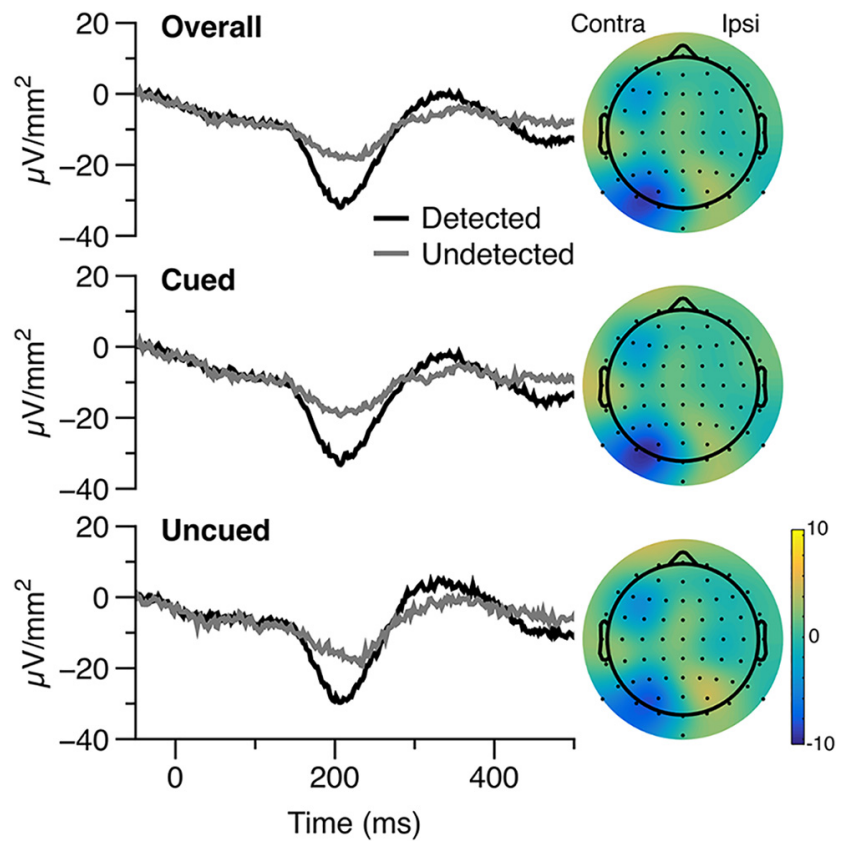

Figure 3. Target-locked ERPs from contralateral focal electrodes. Topographies show the contralateralized difference between responses to detected targets and undetected targets from 165 to 240 ms.

jamini and Hochberg, 1995) revealed no significant differences at any time point between cue-evoked ERPs from trials in which the target was detected and trials in which it was not detected. This was the case at both the contralateral and ipsilateral electrode sites when comparing detected and undetected trials in which the target was cued or uncued (Fig. 4). Because the results of these analyses have implications for the interpretation of our phase dependence analysis, we also report them without any correction for multiple comparisons. It is important to note that, with 256 time points each $(\sim 1.95 \mathrm{~ms}$ per time point $)$ and an alpha level of 0.05 , these analyses would each be expected to produce an average of 12.8 significant differences by chance alone. The same analysis as described above performed at contralateral focal electrodes with no correction for multiple comparisons produced four significantly different time points on cued-target trials (scattered between $-166 \mathrm{~ms}$ and $-152 \mathrm{~ms}$, all $p$-values $>0.028$ ) and five significant differences between time points on uncued-target trials (one at $-420 \mathrm{~ms}$ and four between $-35 \mathrm{~ms}$ and $-5 \mathrm{~ms}$, all 

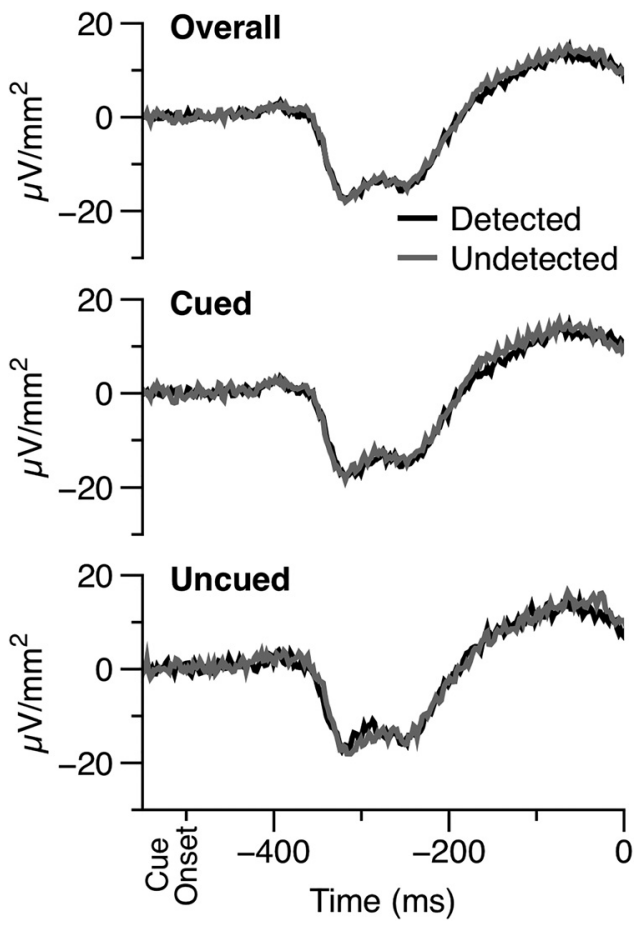

Figure 4. Cue-locked ERPs from contralateral focal electrodes. The time scale is negative to make clear that this is the pretarget period and to facilitate comparison with the timefrequency figures that present data from the same time period.

$p$-values $>0.015)$. The low number of significant time points and the low degree of overlap between these and the times of observed phase dependence, reported below, indicates that the phase results were not driven by differences in cue-evoked ERPs.

\section{Pretarget power}

Comparison of oscillatory power between focal electrodes contralateral and ipsilateral to the cued visual hemifield revealed the expected pretarget alpha amplitude decrease contralateral to the cued side of space. FDR-controlled $t$ tests at every time and frequency revealed that power was significantly lower at contralateral focal electrodes relative to ipsilateral focal electrodes in the range from 10 to $24 \mathrm{~Hz}$ from $-129 \mathrm{~ms}$ until the end of the analysis window at $0 \mathrm{~ms}$ (Fig. 5). This result is consistent with previous studies showing alpha lateralization with attentional allocation (Worden et al., 2000; Sauseng et al., 2005; Thut et al., 2006) and are consistent with participants' shifting their attention covertly to the cued side as intended. Power was also significantly higher contralateral relative to ipsilateral the cued hemifield at $2 \mathrm{~Hz}$ from $-387 \mathrm{~ms}$ until $-125 \mathrm{~ms}$. No power differences were found between the pretrial periods for trials in which the target was detected and those in which it was not detected, consistent with previous results observed with this paradigm (Wyart and TallonBaudry, 2008).

\section{Phase analysis}

The POS metric quantifies the degree to which detected-target versus undetected-target trials cluster at opposing phases of an oscillatory cycle at a particular time and frequency (VanRullen, 2016a; see Materials and Methods for details). This analysis, when performed at focal electrodes contralateral to the location of cued targets, revealed a number of significant time points. There was a cluster of significant phase modulation in the theta frequency range between 4 and $6 \mathrm{~Hz}$ from $-402 \mathrm{~ms}$ to $-279 \mathrm{~ms}$, which had
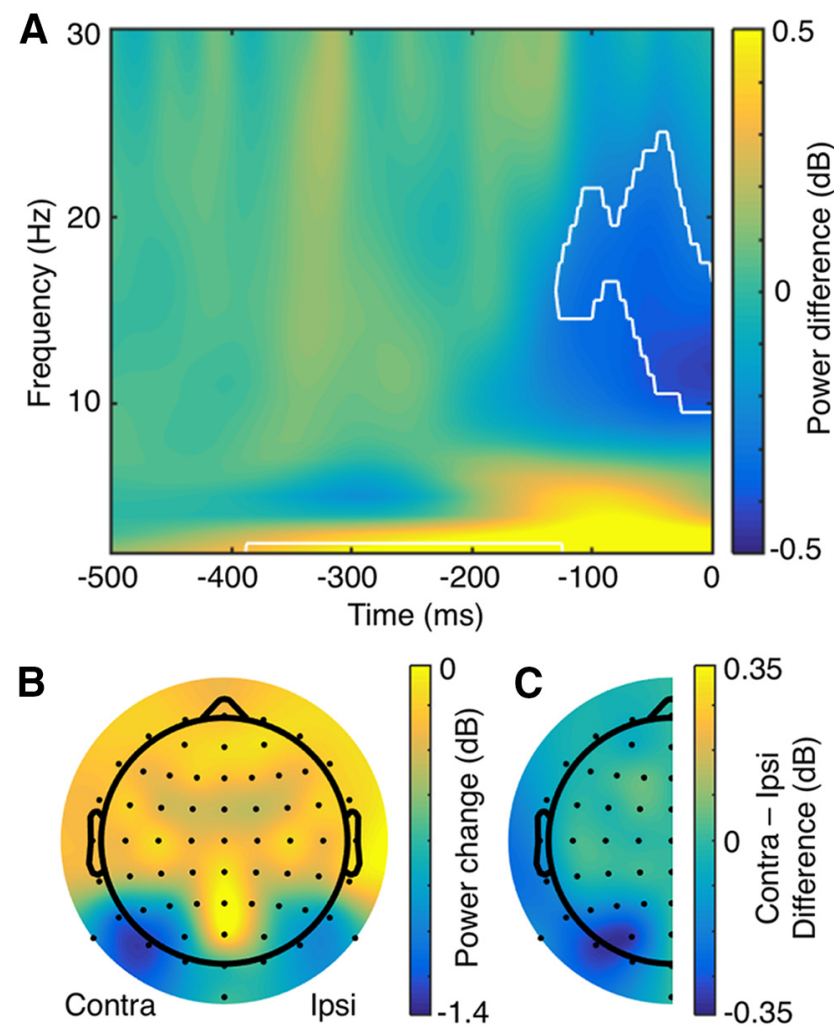

Figure 5. Time-frequency amplitude change showing an attention-related alpha amplitude reduction contralateral to the cued location. $\boldsymbol{A}$, Contralateral minus ipsilateral amplitude difference computed at focal electrodes. Contralateral and ipsilateral are relative to the location of the cue. White boundaries show significant differences corrected for multiple comparisons with cluster-based permutation tests (Groppe et al., 2011). B, Contralateralized scalp topography across the $10-24 \mathrm{~Hz}$ frequency range from -139 until target onset at 0 ms. C, Topography of amplitude difference between contralateral and ipsilateral electrodes.

a $p$-value minimum at the border of the temporal smearing range at $5 \mathrm{~Hz}$ at $-300 \mathrm{~ms}$ (Fig. 6A). A second cluster spanned the alpha-frequency range from 7 to $15 \mathrm{~Hz}$ from $-363 \mathrm{~ms}$ to -240 $\mathrm{ms}$ and had a $p$-value minimum at $11 \mathrm{~Hz}$ at $-316 \mathrm{~ms}$. Other small clusters appeared at other times and frequencies (Fig. 6A).

The same analysis applied to uncued-target trials (Fig. 6B) revealed a cluster of significant theta-phase dependence between 4 and $6 \mathrm{~Hz}$ from the start of the analysis window at $-500 \mathrm{~ms}$ (cue onset) until $-330 \mathrm{~ms}$, with a $p$-value minimum from 4 to $5 \mathrm{~Hz}$ from $-445 \mathrm{~ms}$ to $-377 \mathrm{~ms}$. The second cluster was between $11 \mathrm{~Hz}$ and $15 \mathrm{~Hz}$ from $-346 \mathrm{~ms}$ to $-302 \mathrm{~ms}$, with a $p$-value minimum at $14 \mathrm{~Hz}$ at $-318 \mathrm{~ms}$. Interestingly, the temporal and frequency properties of this second cluster overlap closely with the alpha-frequency cluster found for the cued-target condition. There was also considerable overlap between the $4-6 \mathrm{~Hz}$ thetafrequency cluster observed here and the $4-6 \mathrm{~Hz}$ theta-frequency cluster observed for the cued-target condition, although the cluster revealed here for uncued trials began considerably earlier, with its period of minimum $p$-value beginning before the significant period began in the cued-target condition. This result may suggest stronger phase dependence for uncued stimuli in the theta-frequency range. An important caveat, however, is that, although $p$-values were lower in the cued-target condition, this result might have arisen merely because there were more trials in the cued than in the uncued condition. To address this possibility, we repeated the above analysis after subsampling an equal number of trials in all conditions (detected cued targets, unde- 

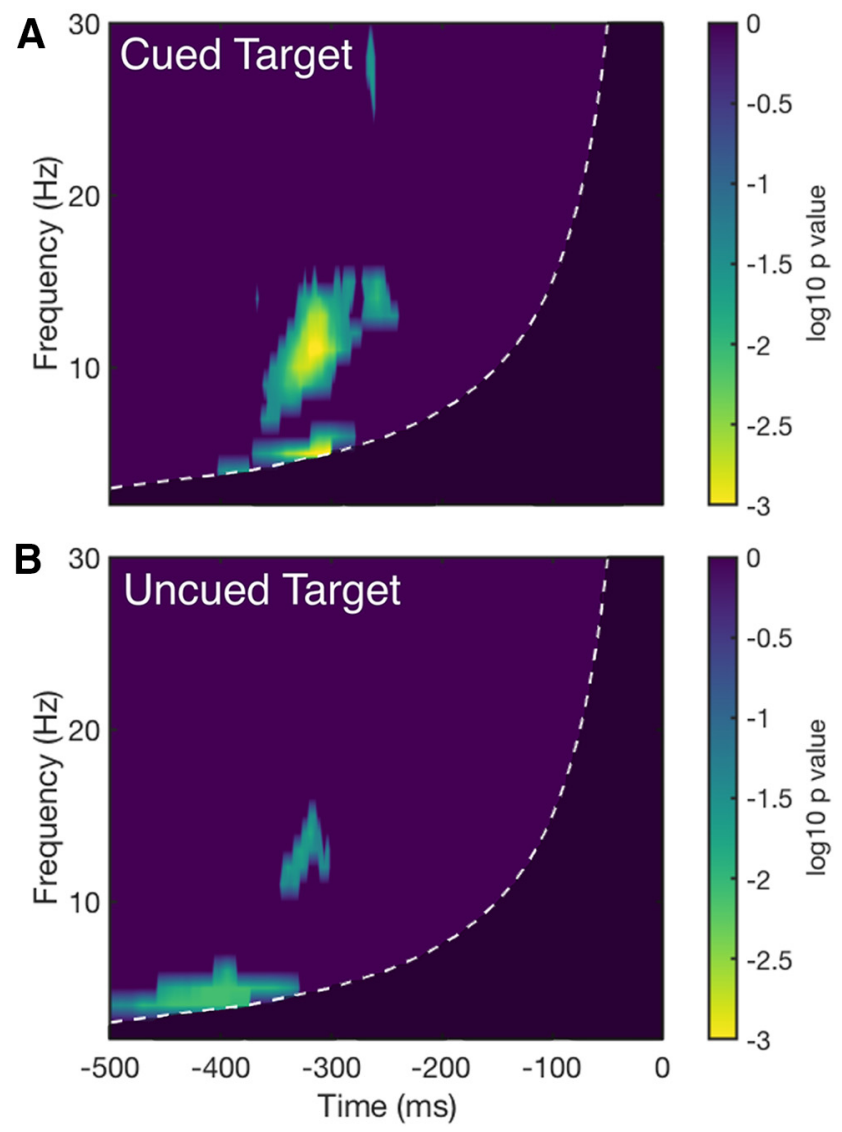

Figure 6. Time-frequency maps of $p$-values resulting from pretrial POS analysis across frequency and time thresholded to show only significant effects $(p<0.05)$. $\boldsymbol{A}$, Cued-target condition. $\boldsymbol{B}$, Uncued-target condition. Dashed white lines represent the edge of the temporalsmearing boundary. Times to the right of this line (indicated in dark purple) were not analyzed due to the possibility that values in this range might have been influenced by posttarget activity.

tected cued targets, detected uncued targets, and undetected uncued targets). This analysis also yielded significant results, but with $p$-values lower in the uncued-target condition. Because it is inappropriate to interpret $p$-values in terms of strength of evidence, we next compared the influence of prestimulus oscillations on target detection directly between cued-target and uncued-target trials.

To compare the influence of prestimulus oscillatory phase between cued-target and uncued-target trials for the frequency ranges identified above, we performed an analysis in which we sorted trials into nine nonoverlapping bins of equal width based on the phase of contralateral prestimulus oscillations at a particular time and frequency and calculated the detection rate for the trials within each phase bin. The optimally performing bins were then aligned, as described in previous studies (Busch et al., 2009; Busch and VanRullen, 2010). The bin with the highest detection rate was deemed the $0^{\circ}$ bin and the other bins maintained their relationship relative to this bin. If a relationship between phase and performance is present, then bins neighboring the optimal bin should show monotonically decreasing performance with increasing circular distance from the optimal bin (after excluding the optimal bin from analysis because performance in this bin is superior by definition). In contrast, if there is no phasic relationship in the data, then the "optimal" bin should exhibit superior performance by chance only and the neighboring bins should show no systematic pattern of performance. Phase binning and optimal bin alignment were performed at each time and frequency within the relevant ranges and then averaged across time and frequency. We performed a repeated-measures ANOVA on detection rates across each phase bin and cue condition, excluding the "optimal" phase bin. Greenhouse-Geisser correction was applied when Mauchly's test indicated that the assumption of sphericity had been violated.

We first compared phase dependence between cued and uncued trials in the range in which their pretrial effects overlapped, from 4 to $6 \mathrm{~Hz}$, between $-402 \mathrm{~ms}$ and $-330 \mathrm{~ms}$. This produced a significant main effect of phase $\left(F_{(2.41,60.25)}=36.66, p<0.001\right.$, $\left.\eta^{2}=0.60\right)$. The main effect of cue condition was not significant $\left(F_{(1,25)}=0.80, p=0.381, \eta^{2}=0.03\right)$, but there was a significant interaction between cue condition and phase $\left(F_{(3.82,95.52)}=4.88\right.$, $\left.p=0.001, \eta^{2}=0.16\right)$, indicating a larger effect of theta phase in the uncued condition than in the cued condition (Fig. 7A). The effect of $4-6 \mathrm{~Hz}$ theta phase on detection of cued targets accounted for $10.57 \%$ of the overall detection rate compared with $19.74 \%$ for uncued targets. These percentages are likely to be underestimates due to exclusion of the optimal phase bin, as described above.

The same analysis performed on the overlap between the higherfrequency activity from the cued and uncued conditions, from 11 to $15 \mathrm{~Hz}$, between $-346 \mathrm{~ms}$ and $-302 \mathrm{~ms}$ revealed a significant main effect of phase $\left(F_{(3.06,76.60)}=65.18, p<0.001, \eta^{2}=0.72\right)$, but no significant main effect of cue condition $\left(F_{(1,25)}=0.13, p=\right.$ $\left.0.725, \eta^{2}<0.01\right)$ and no significant interaction $\left(F_{(2.66,66.44)}=\right.$ $\left.0.94, p=0.420, \eta^{2}=0.04\right)$, indicating an effect of alpha phase that was the same on cued-target and uncued-target trials (Fig. $7 B)$. The effect of $11-15 \mathrm{~Hz}$ alpha phase on detection of cued targets accounted for $11.47 \%$ of the overall detection rate compared with $14.58 \%$ for uncued targets. To control for the possibility of different trial numbers contributing to differences between the conditions, we repeated these analyses with equal numbers of randomly selected trials in all conditions. All of the significant effects identified in the original analysis were replicated in this additional trial-balanced analysis.

When oscillatory amplitude is close to zero, phase values become difficult to estimate, so it is possible for amplitude differences between conditions to produce spurious differences in phase metrics. It should be noted that the phase differences reported here for detected versus undetected targets (Fig. 6) and the differences in phase dependence for cued-target versus uncuedtarget trials (Fig. 7) occurred at different times and frequencies from the significant power reduction shown in Figure 5. Therefore, any difference in phase metrics is unlikely to be related to differences in power between the conditions.

High intertrial phase consistency (ITPC) has the potential to mask the effects of phase on perception. Therefore, it is important to rule out the possibility that differences in detection between conditions as a function of phase could be driven by differences in ITPC in the pretarget period. ITPC is known to be inflated by low trial numbers (Cohen, 2014), so for the following analyses, we selected random subsamples of trials in each condition to equate trial numbers across all the conditions. These analyses were performed with FDR-corrected $t$ tests (except where noted) at every time and frequency outside of the temporal smearing region.

We first compared ITPC at focal electrodes contralateral to the target for detected-target versus undetected-target trials separately for cued-target and for uncued-target trials to control for the effects of ITPC in the POS analysis above (Fig. 6). This analysis revealed no significant differences in either the cued-target or uncued-target trials at any time or frequency (cued: $p$-values $>$ 

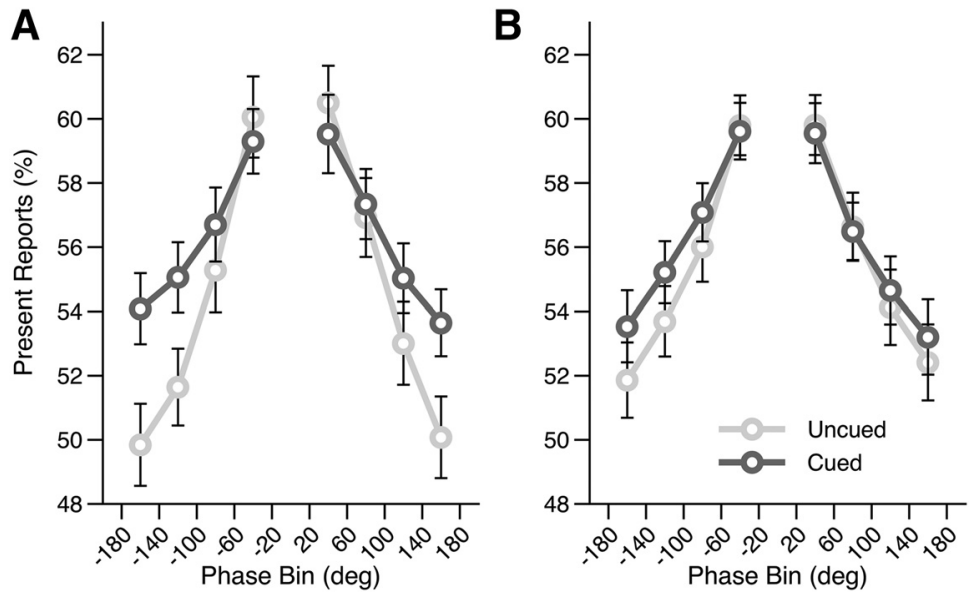

Figure 7. Comparison of phasic differences in detection-rate between the cued and uncued conditions. Phase bins represent phase relative to the bin showing optimal performance, which was aligned to the center phase bin $\left(-20^{\circ}\right.$ to $\left.20^{\circ}\right)$. $A$, Detection rates binned by $4-6 \mathrm{~Hz}$ phase from $-402 \mathrm{~ms}$ to $-330 \mathrm{~ms}$. $B$, Detection rates binned by $11-15 \mathrm{~Hz}$ phase from $-346 \mathrm{~ms}$ to -302 ms. Error bars indicate within-participant SEM (Cousineau, 2005; Morey, 2008).

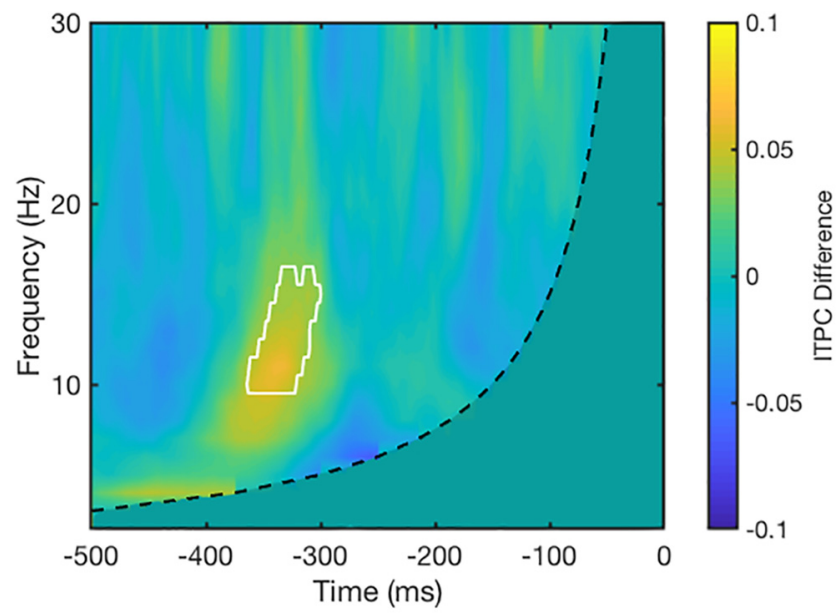

Figure 8. Difference in ITPC between cued-target and uncued-target trials. Dashed black line represents the edge of the temporal-smearing boundary, with the dark gray section representing times that could not be analyzed due to the possibility of contamination from posttarget events. Warm colors represent higher ITPC on cued-target trials; cool colors represent higher ITPC on uncued-target trials. The white border outlines the period of significant difference as determined with cluster-based permutation tests (Groppe et al., 2011).

0.717 ; uncued: $p$-values $>0.601)$. Therefore, differences in ITPC are unlikely to have driven the effects observed in the POS analysis. Further reanalyses correcting for multiple comparisons with clusterbased permutation tests (Groppe et al., 2011) produced the same results (cued: cluster $p$-values $>0.999$; uncued: cluster $p$-values $>0.159$ ).

We also compared ITPC between cued-target and uncuedtarget trials, collapsing across detection conditions, to examine the impact of ITPC on phase dependence differences between the conditions. This analysis revealed no significant difference at any time or frequency when correcting for multiple-comparisons using FDR ( $p$-values $>0.227$ ). In contrast, reanalysis with multiple comparisons controlled using cluster-based permutation tests revealed a cluster of significantly stronger ITPC in the cuedtarget condition than in the uncued-target condition (cluster $p=$ 0.048, from 10 to $16 \mathrm{~Hz}$, between $-363 \mathrm{~ms}$ and $-301 \mathrm{~ms}$; Fig. 8). This effect overlaps in time and frequency with the alpha- frequency POS results reported above and suggests that the alpha-frequency phase dependence of perception described earlier for the cued-target condition (Fig. 7B) might have been underestimated relative to the uncued-target condition. Importantly, however, there was no significant ITPC difference at the times and frequencies of the theta-frequency phase effect. Furthermore, focusing exclusively on the time-frequency range of the theta phase effect showed that average ITPC was not significantly different between detectedtarget and undetected-target trials across that range $(4-6 \mathrm{~Hz}$, between -403 and $-330 \mathrm{~ms} ; t_{(25)}=1.22, p=0.233$, Cohen's $\mathrm{d}=0.24)$. ITPC differences between the conditions therefore cannot explain the theta-frequency difference in phase dependence between cued-target and uncuedtarget trials (Fig. 7A).

\section{Discussion}

Few studies have attempted to differentiate the neural oscillations that relate to phasic modulation of perception at attended and unattended locations, making it unclear whether oscillatory effects on perception are intrinsic to the functioning of sensory cortex or if they are driven by top-down mechanisms such as attention. Here, we investigated whether the phase of prestimulus oscillations modulates the processing of stimuli outside of the focus of attention, and whether differences exist in the phase dependence of visual processing between attended and unattended stimuli. We predicted that, if unattended locations are not subject to phasic modulation of stimulus processing (Busch and VanRullen, 2010), then we would observe no relationship between prestimulus phase and uncued-target detection at any frequency. Alternatively, if unattended locations are subject to phasic modulation of stimulus processing, then we expected one of two outcomes. We might observe the same phase dependence characteristics at both attended and unattended locations, indicating a perceptual sampling process that is independent of attention. Alternatively, we might observe phase dependence for both attended and unattended locations that differs between locations. This would indicate a sampling process that is affected by attention.

Our results confirm that processing of stimuli outside of the focus of attention is subject to phasic modulation by ongoing oscillations recorded contralateral to the location of the stimulus. Specifically, for both cued and uncued targets, we observed that target detection fluctuated with the phase of both an $\sim 5 \mathrm{~Hz}$ theta rhythm and a broad high-alpha frequency rhythm (from 11 to 15 $\mathrm{Hz}$ ). These results are in contrast to previous work (Busch and VanRullen, 2010) that did not account for the location of the target by contralateralizing the data and found no phase dependence for uncued stimuli. We also found that pretrial theta phase modulated the detection of unattended stimuli almost twice as strongly as it did attended stimuli, suggesting the allocation of spatial attention produces a relative disengagement from ongoing theta-frequency sampling. In contrast, alpha-phase dependence was no different for cued and uncued stimuli, consistent with suggestions that the phase of posterior alpha rhythms instantiates an ongoing perceptual sampling process rather than being a sampling mechanism that is engaged when attention is 
allocated (VanRullen, 2016b). We hesitate to place too much emphasis on the apparent similarity of alpha-phase dependence for cued-target and uncued-target trials, however, due to ITPC differences that confound the phase dependence analysis at alpha frequencies.

The pretarget period examined in our phase dependence analysis was also the period after the spatial cue that directed participants to attend to one location or the other. To conclude that the phase dependence effects that we observed reflect the influence of ongoing endogenous oscillations on perception, it is important to rule out that such phase effects might be driven instead by differences in the evoked response to the cue for detected-target and undetected-target trials. Any differences in evoked responses may appear as differences in phase or amplitude when decomposed into the time-frequency domain without necessarily being caused by a change in endogenous oscillations (Sauseng et al., 2007). Such cue-related ERP differences between detected-target and undetected-target trials are unlikely given that the stimuli in these conditions were physically identical. Indeed, we showed that cue-evoked ERPs recorded at exactly the times and electrodes that were included in the phase analysis showed no significant differences between detected-target and undetected-target trials. We can therefore be confident that the phase dependence of target detection observed during this period reflected actual differences in pretrial phase between detected and undetected targets and not merely differences in the cue-evoked ERP.

The phase-dependent detection that we observed at alpha frequencies is consistent with previous results demonstrating posterior alpha frequency sampling when to-be-detected stimuli are presented at a known, attended location (Mathewson et al., 2009; Sherman et al., 2016). Here, we extend these results by demonstrating that alpha-phase dependence is of equal magnitude for stimuli appearing at attended and unattended locations, consistent with the suggestion that alpha phase modulates perceptual processing independently of attention (VanRullen, 2016b). This is a counterintuitive result given the well established finding that the strength of alpha oscillations (i.e., their amplitude) is relatively decreased contralateral to the location of spatial attention (Worden et al., 2000; Sauseng et al., 2005; Kelly et al., 2006; Thut et al., 2006), and the degree of attention-related alpha amplitude lateralization predicts behavioral performance (Händel et al., 2011; van Diepen et al., 2016). Alpha oscillations have been strongly implicated in the inhibition of neural processing, with increased alpha amplitude associated with increased inhibition in sensory cortices (Goldman et al., 2002; Romei et al., 2008; Lange et al., 2013). Therefore, one might expect the degree of alpha phase dependence to be related to alpha amplitude (Jensen et al., 2012, 2014). As noted above, however, we do not rely too heavily on the finding of comparable phase dependence for cued-target and uncued-target trials for two reasons. First, as noted above, the ITPC analysis revealed a difference between cued-target and uncued-target trials in the degree of pretrial phase clustering at alpha frequencies, which might have masked the cued-target phase dependence to some degree. Second, the time range over which we observed pretrial alpha-power lateralization did not overlap with the time range over which we observed pretrialalpha phase opposition. Therefore, the potential interrelation between alpha amplitude and alpha-phase dependence requires further empirical appraisal.

Recent work has suggested that the monitoring of multiple locations by spatial attention may be instantiated by an $\sim 7-8 \mathrm{~Hz}$ attentional rhythm that samples different locations on successive oscillatory cycles, effectively sampling each location at a frequency that is proportional to $7-8 \mathrm{~Hz}$ sampling divided by the number of locations (Fries, 2009, 2015; Holcombe and Chen, 2013; Landau et al., 2015). Our results showing a reduced influence of theta oscillations for the perception of attended stimuli relative to unattended stimuli suggest that this sampling process need not necessarily be divided evenly across all possible target locations. Rather, theta sampling might be subject to top-down control, not only for selection of the specific locations to which it is applied, but also for the degree to which it is applied at each location. Theta rhythmic sampling could be applied asymmetrically across locations based on the likelihood of a target appearing at each location.

Our results in human observers are strikingly consistent with the findings of a recent experiment that used electrocorticography in monkeys (Spyropoulos et al., 2017). The investigators trained two macaques to perform a visual attention task and calculated Granger causal influence in the theta-frequency band between signals from different regions of visual cortex. They found that Granger causal influence in the theta band was reduced when attention was directed to the stimulus contralateral to the recording site, but was maintained when attention was directed to an ipsilateral stimulus. This result might provide a neuronal mechanism for the present finding of an attention-related disengagement of theta-frequency sampling. Decoupling neural signal transmission from low-frequency oscillations could potentially result in a more continuous mode of sensory processing that is less subject to processing delays produced by regular periods of inhibition (Schroeder and Lakatos, 2009).

Previous studies have shown theta-frequency oscillations in behavioral responses to targets at unattended locations that are not present for attended targets (Landau and Fries, 2012; Fiebelkorn, Saalmann, and Kastner, 2013). Attempts have been made to link these effects to neural oscillations by demonstrating, for example, a theta-frequency pattern of performance disruption produced by transcranial magnetic stimulation contralateral to an unattended location (Dugué et al., 2016). Although such approaches are highly suggestive, they are ultimately inconclusive about the neural source of such rhythmic effects. Our findings go beyond this work by showing a significant association between detection performance and the phase of prestimulus oscillations and by revealing a reliable modulation of this relationship by covert spatial attention.

In summary, we have demonstrated that stimulus detection at unattended locations is subject to phasic modulation by prestimulus neural oscillations at cortical sites that process early stimulus-related information. Phase-dependent perception at alpha frequencies seems to be independent of attention, consistent with a perceptual sampling process (VanRullen, 2016b). In contrast, phase dependence at theta frequencies is most strongly associated with sampling of unattended locations, modulating performance by almost $20 \%$, whereas attended locations are less influenced by theta phase. Our findings suggest that attentional allocation is associated with a disconnection from ongoing theta sampling in visual cortex (Landau et al., 2015). Future empirical and theoretical work should be directed to understanding the mechanisms by which such disengagement from an ongoing sampling process occurs.

\section{References}

Başar E, Başar-Eroglu C, Karakaş S, Schürmann M (2001) Gamma, alpha, delta, and theta oscillations govern cognitive processes. International Journal of Psychophysiology 39:241-248. CrossRef Medline

Benjamini Y, Hochberg Y (1995) Controlling the false discovery rate: a practical and powerful approach to multiple testing. Journal of the Royal Statistical Society Series B (Methodological) 57:289-300.

Brainard DH (1997) The psychophysics toolbox. Spat Vis 10:433-436. CrossRef Medline 
Busch NA, VanRullen R (2010) Spontaneous EEG oscillations reveal periodic sampling of visual attention. Proc Natl Acad Sci U S A 107:1604816053. CrossRef Medline

Busch NA, Dubois J, VanRullen R (2009) The phase of ongoing EEG oscillations predicts visual perception. J Neurosci 29:7869-7876. CrossRef Medline

Buzsáki, G (2006) Rhythms of the brain. New York, NY: OUP.

Buzsáki G, Draguhn A (2004) Neuronal oscillations in cortical networks. Science 304:1926-1929. CrossRef Medline

Cohen, MX (2014) Analyzing neural time series data: theory and practice. Cambridge, MA: MIT.

Cousineau D (2005) Confidence intervals in within-subject designs: a simpler solution to loftus and Masson's method. Tutorials in Quantitative Methods for Psychology 1:42-45. CrossRef

Delorme A, Makeig S (2004) EEGLAB: an open source toolbox for analysis of single-trial EEG dynamics including independent component analysis. J Neurosci Methods 134:9-21. CrossRef Medline

Drewes J, VanRullen R (2011) This is the rhythm of your eyes: the phase of ongoing electroencephalogram oscillations modulates saccadic reaction time. J Neurosci 31:4698-4708. CrossRef Medline

Dugué L, Marque P, VanRullen R (2011) The phase of ongoing oscillations mediates the causal relation between brain excitation and visual perception. J Neurosci 31:11889-11893. CrossRef Medline

Dugué L, Roberts M, Carrasco M (2016) Attention reorients periodically. Curr Biol 26:1595-1601. CrossRef Medline

Eimer, M (2014) The time course of spatial attention: Insights from eventrelated brain potentials. In: The Oxford handbook of attention (Nobre AC, Kastner S, eds), pp 289-317. Oxford, UK: Oxford University Press.

Fiebelkorn IC, Saalmann YB, Kastner S (2013) Rhythmic sampling within and between objects despite sustained attention at a cued location. Curr Biol 23:2553-2558. CrossRef Medline

Fiebelkorn IC, Snyder AC, Mercier MR, Butler JS, Molholm S, Foxe JJ (2013) Cortical cross-frequency coupling predicts perceptual outcomes. Neuroimage 69:126-137. CrossRef Medline

Fries P (2009) Neuronal gamma-band synchronization is a fundamental process in cortical computation. Ann Rev Neurosci 32:209-224. CrossRef Medline

Fries P (2015) Rhythms for cognition: communication through coherence. Neuron 88:220-235. CrossRef Medline

Goldman RI, Stern JM, Engel J Jr, Cohen MS (2002) Simultaneous EEG and fMRI of the alpha rhythm. Neuroreport 13:2487-2492. CrossRef Medline

Groppe DM, Urbach TP, Kutas M (2011) Mass univariate analysis of eventrelated brain potentials/fields I: a critical tutorial review. Psychophysiology 48:1711-1725. CrossRef Medline

Händel BF, Haarmeier T, Jensen O (2011) Alpha oscillations correlate with the successful inhibition of unattended stimuli. J Cogn Neurosci 23: 2494-2502. CrossRef Medline

Holcombe AO, Chen WY (2013) Splitting attention reduces temporal resolution from $7 \mathrm{hz}$ for tracking one object to $<3 \mathrm{hz}$ when tracking three. $\mathrm{J}$ Vis 13:12. CrossRef Medline

Jensen O, Bonnefond M, VanRullen R (2012) An oscillatory mechanism for prioritizing salient unattended stimuli. Trends Cogn Sci 16:200-206. CrossRef Medline

Jensen O, Gips B, Bergmann TO, Bonnefond M (2014) Temporal coding organized by coupled alpha and gamma oscillations prioritize visual processing. Trends Neurosci 37:357-369. CrossRef Medline

Kelly SP, Lalor EC, Reilly RB, Foxe JJ (2006) Increases in alpha oscillatory power reflect an active retinotopic mechanism for distracter suppression during sustained visuospatial attention. J Neurophysiol 95:3844-3851. CrossRef Medline

Kleiner M, Brainard D, Pelli D, Ingling A, Murray R (2007) What's new in Psychtoolbox-3? Perception 36:1-16. Available at https://nyuscholars. nyu.edu/en/publications/whats-new-in-psychtoolbox-3.

Landau AN, Fries P (2012) Attention samples stimuli rhythmically. Curr Biol 22:1000-1004. CrossRef Medline

Landau AN, Schreyer HM, van Pelt S, Fries P (2015) Distributed attention is implemented through theta-rhythmic gamma modulation. Curr Biol 25: 2332-2337. CrossRef Medline

Lange J, Oostenveld R, Fries P (2013) Reduced occipital alpha power indexes enhanced excitability rather than improved visual perception. J Neurosci 33:3212-3220. CrossRef Medline
Mathewson KE, Gratton G, Fabiani M, Beck DM, Ro T (2009) To see or not to see: prestimulus $\alpha$ phase predicts visual awareness. J Neurosci 29:27252732. CrossRef Medline

Morey R (2008) Confidence intervals from normalized data: a correction to Cousineau (2005). Tutorial in Quantitative Methods for Psychology 4:61-64. CrossRef

Morris SB, DeShon RP (2002) Combining effect size estimates in metaanalysis with repeated measures and independent-groups designs. Psychol Methods 7:105-125. CrossRef Medline

Oostenveld R, Praamstra P (2001) The five percent electrode system for high-resolution EEG and ERP measurements. Clin Neurophysiol 112: 713-719. CrossRef Medline

Perrin F, Pernier J, Bertrand O, Echallier JF (1989) Spherical splines for scalp potential and current density mapping. Electroencephalogr Clin Neurophysiol 72:184-187. CrossRef Medline

Pestilli F, Carrasco M (2005) Attention enhances contrast sensitivity at cued and impairs it at uncued locations. Vision Res 45:1867-1875. CrossRef Medline

Romei V, Brodbeck V, Michel C, Amedi A, Pascual-Leone A, Thut G (2008) Spontaneous fluctuations in posterior $\alpha$-band EEG activity reflect variability in excitability of human visual areas. Cereb Cortex 18:2010-2018. CrossRef Medline

Sauseng P, Klimesch W, Stadler W, Schabus M, Doppelmayr M, Hanslmayr S, Gruber W, Birbaumer N (2005) A shift of visual spatial attention is selectively associated with human EEG alpha activity. Eur J Neurosci 22: 2917-2926. CrossRef Medline

Sauseng P, Klimesch W, Gruber WR, Hanslmayr S, Freunberger R, Doppelmayr M (2007) Are event-related potential components generated by phase resetting of brain oscillations? A critical discussion. Neuroscience 146:1435-1444. CrossRef Medline

Schroeder CE, Lakatos P (2009) Low frequency neuronal oscillations as instruments of sensory selection. Trends Neurosci 32:9-18. CrossRef Medline

Sherman MT, Kanai R, Seth AK, VanRullen R (2016) Rhythmic influence of top-down perceptual priors in the phase of prestimulus occipital alpha oscillations. J Cogn Neurosci 28:1318-1330. CrossRef Medline

Spyropoulos G, Bosman CA, Fries P (2017) A theta rhythm in awake macaque visual cortex and its attentional modulation. BioRxiv. Advance online publication. Accessed on November 2017. CrossRef

Stouffer SA, Suchman EA, DeVinney LC, Star SA, Williams RM Jr (1949) The American soldier, Vol 1: adjustment during Army life. Princeton, NJ: Princeton University.

Tallon-Baudry C, Bertrand O (1999) Oscillatory gamma activity in humans and its role in object representation. Trends Cogn Sci 3:151-162. Medline

Thut G, Nietzel A, Brandt SA, Pascual-Leone A (2006) Alpha-band electroencephalographic activity over occipital cortex indexes visuospatial attention bias and predicts visual target detection. J Neurosci 26:9494-9502. CrossRef Medline

Tyler CW (1997) Colour bit-stealing to enhance the luminance resolution of digital displays on a single pixel basis. Spat Vis 10:369-377. CrossRef Medline

van Diepen RM, Miller LM, Mazaheri A, Geng JJ (2016) The role of alpha activity in spatial and feature-based attention. eNeuro 3: pii: ENEURO. 0204-16.2016. CrossRef Medline

VanRullen R (2016a) How to evaluate phase differences between trial groups in ongoing electrophysiological signals. Front Neurosci 10:426. CrossRef Medline

VanRullen R (2016b) Perceptual cycles. Trends Cogn Sci 20:723-735. CrossRef Medline

Watson AB, Pelli DG (1983) Quest: a bayesian adaptive psychometric method. Percept Psychophys 33:113-120. CrossRef Medline

Worden MS, Foxe JJ, Wang N, Simpson GV (2000) Anticipatory biasing of visuospatial attention indexed by retinotopically specific $\alpha$-band electroencephalography increases over occipital cortex. J Neurosci 20: RC63. Medline

Wyart V, Tallon-Baudry C (2008) Neural dissociation between visual awareness and spatial attention. J Neurosci 28:2667-2679. CrossRef Medline

Zeki SM (1978) Functional specialisation in the visual cortex of the rhesus monkey. Nature 274:423-448. CrossRef Medline 